\title{
The Behavioral Sequelae of Social Defeat Require Microglia and Are Driven by Oxidative Stress in Mice
}

\author{
Michael L. Lehmann,* Thaddeus K. Weigel,^ Chelsie N. Poffenberger, and $\odot$ Miles Herkenham \\ Section on Functional Neuroanatomy, Intramural Research Program, National Institute of Mental Health, National Institutes of Health, Bethesda, Maryland \\ 20892
}

Chronic social defeat (CSD) in male mice can produce anxiety and aberrant socialization. Animals susceptible to CSD show activation of microglia, which have elevated levels of oxidative stress markers. We hypothesized that microglia and reactive oxygen species (ROS) production contribute to the CSD stress-induced changes in affective behavior. First, we selectively depleted microglia (99\%) by administering the CSF1R (colony-stimulating factor 1 receptor) antagonist PLX5622 before and during the $14 \mathrm{~d}$ CSD procedure. Microgliadepleted mice in contrast to nondepleted mice were protected from the stress effects measured by light/dark and social interaction tests. ROS production, measured histochemically following dihydroethidium administration, was elevated by CSD, and the production was reduced to basal levels in mice lacking microglia. The deleterious stress effects were also blocked in nondepleted mice by continuous intracerebral administration of $N$-acetylcysteine (NAC), a ROS inhibitor. In a second experiment, at the end of the CSD period, PLX5622 was discontinued to allow microglial repopulation. After $14 \mathrm{~d}$, the brain had a full complement of newly generated microglia. At this time, the mice that had previously been protected now showed behavioral deficits, and their brain ROS production was elevated, both in all brain cells and in repopulated microglia. NAC administration during repopulation prevented the behavioral decline in the repopulated mice, and it supported behavioral recovery in nondepleted stressed mice. The data suggest that microglia drive elevated ROS production during and after stress exposure. This elevated ROS activity generates a central state supporting dysregulated affect, and it hinders the restoration of behavioral and neurochemical homeostasis after stress cessation.

Key words: inflammation; microglia; neuroimmune; oxidative stress; psychosocial stress; ROS

\section{Significance Statement}

Chronic psychosocial stress is associated with psychiatric disorders such as depression and anxiety. Understanding the details of CNS cellular contributions to stress effects could lead to the development of intervention strategies. Inflammation and oxidative stress are positively linked to depression severity, but the cellular nature of these processes is not clear. The chronic social defeat (CSD) paradigm in mice produces mood alterations and microglial activation characterized by elevated reactive oxygen species (ROS) production. The depletion of microglia or ROS inhibition prevented adverse stress effects. Microglial repopulation of the brain post-CSD reintroduced adverse stress effects, and ROS inhibition in this phase protected against the effects. The results suggest that stress-induced microglial ROS production drives a central state that supports dysregulated affective behavior.

\section{Introduction}

Microglia are highly dynamic cells with unique properties. After migration into the brain in early gestation (Ginhoux et al., 2010),

\footnotetext{
Received Jan. 22, 2019; revised April 26, 2019; accepted May 4, 2019.

Author contributions: M.L.L. and M.H. designed research; M.L.L., T.K.W., and C.N.P. performed research; M.L.L. and T.K.W. analyzed data; M.H. wrote the paper.

*M.L.L. and T.K.W. are co-first authors.

The work was supported by the Intramural Research Program, National Institute of Mental Health Grant ZIA-MH001090. We thank Plexxikon for the gift of PLX5622, and Lindsay Appleman for technical assistance.

The authors declare no competing financial interests.

Correspondence should be addressed to Miles Herkenham at herkenh@mail.nih.gov.

T.K. Weigel's present address: Graduate Program in Neuroscience, School of Medicine, University of Virginia, Charlottesville, VA 22908.
}

they homeostatically sustain their numbers throughout life by local self-renewal (Ajami et al., 2007; Tay et al., 2017b). As the resident innate immune cells of the brain, they share many features with bone marrow-derived monocytes, though they have distinguishing gene expression profiles (Bennett et al., 2016). Microglia continuously surveil the parenchyma (Nimmerjahn et al., 2005); support brain development and aging, synaptic connections, and neuronal function (Prinz et al., 2014; Tay et al., 2017a; Hammond et al., 2018); and contribute to the regulation of neurodegenerative (Keren-Shaul et 
al., 2017; Butovsky and Weiner, 2018) and psychiatric diseases (Prinz and Priller, 2014; Hammond et al., 2018).

Microglia are activated by immune challenges and also by nonimmune challenges such as psychological stress, wherein the degree and profile of activation are influenced by the type, duration, and severity of the stressor (Walker et al., 2013). We have used chronic social defeat (CSD; Lehmann et al., 2016), a form of psychosocial stress that has validity (Pollak et al., 2010) for modeling the relationship in humans between stressful life events and the development of anxiety and depression (Kendler and Gardner, 2010). Animals that are behaviorally susceptible to the deleterious effects of CSD show microglial activation and bloodbrain barrier (BBB) breakdown (Menard et al., 2017; Lehmann et al., 2018). Microglia in this context have gene ontology profiles indicating elevated inflammation, phagocytosis, matrix remodeling, and production of reactive oxygen species (ROS; Lehmann et al., 2018). Elevated ROS production might drive dynamic changes in microglia during CSD (Minakami and Sumimotoa, 2006; Rojo et al., 2014; ElAli and Rivest, 2016), and it might modify activity in the neural circuitry associated with emotional responses in stressed animals (Liu et al., 2003; Seo et al., 2012; Popa-Wagner et al., 2013). In both animals and humans, psychological stress has been shown to trigger oxidative stress in the brain (Seo et al., 2012; Schiavone et al., 2013; Salim, 2014; Ibi et al., 2017), which is linked to psychiatric disorders (Ng et al., 2008; Black et al., 2015). We therefore asked (1) whether microglia significantly contribute to the deleterious psychological and physical effects of CSD and (2) whether oxidative stress associated with the microglial response drives the behavioral outcomes. We approached these questions by first asking whether the elimination of microglia during the CSD would impart protection against its behavioral effects. To achieve this, we administered PLX5622, a CSF1R (colony-stimulating factor 1 receptor) antagonist that reversibly eliminates microglia (Dagher et al., 2015). Second, we addressed the role played by oxidative stress in mice in which ROS was blocked by the administration of $\mathrm{N}$ acetylcysteine (NAC), a ROS inhibitor (Bavarsad Shahripour et al., 2014), during the stress exposure. We found that microglial elimination and ROS inhibition both protected against deleterious stress effects.

Upon discontinuation of PLX5622, microglia rapidly repopulate the brain through a process of self-renewal of surviving cells (Epelman et al., 2014; Elmore et al., 2015; Huang et al., 2018). To examine the residual effects of stress exposure in microgliadepleted mice, we allowed the repopulation to occur and then assayed for behavior and ROS status. Surprisingly, behavioral deficits emerged in the microglia-repopulated mice, paralleled by elevated ROS levels. ROS blockade by NAC in the repopulation period prevented the deficits. Together, the data indicate that microglia drive changes in affective behavior during and after exposure to psychosocial stress through changes in oxidative stress.

\section{Materials and Methods \\ Animals}

All procedures were approved by the National Institute of Mental Health Institutional Animal Care and Use Committee and conducted in accordance with the National Institutes of Health guidelines. Behavioral experiments were performed using male $\mathrm{CD}-1$ retired breeder mice and 8- to 10-week-old male C57BL/6N mice (Charles River Laboratories). Cx3cr1 ${ }^{\text {wt } / 8 f p}$ mice, $8-10$ weeks of age (model B6.129P-Cx3crr $1^{\text {tm ILitt/J }}$ backcrossed onto a C57BL/6N background, The Jackson Laboratory) were used for tracking microglial depletion and repopulation. All test animals were group housed in pathogen-free conditions in a $12 \mathrm{~h}$ light/ dark (L/D) cycle with lights off at 9:00 A.M. Food and water were provided ad libitum. Behavioral testing was performed in the dark phase under dim lighting.

\section{Compounds}

Experimental groups were fed the CSF1R inhibitor PLX5622, which was provided by Plexxikon and formulated in AIN-76A standard chow at 1200 mg/kg by Research Diets. Control animals were fed AIN-76A standard chow (Research Diets).

\section{Chronic social defeat}

CSD was used to model the effects of chronic psychosocial stress in mice. As previously described (Lehmann and Herkenham, 2011), an experimental C57BL/6 mouse was housed for $14 \mathrm{~d}$ in the home cage (HC) of an aggressive male CD-1 mouse with a perforated transparent acrylic partition separating the mice. The partition was removed for 5 min each day to allow agonistic encounters between the mice. Unstressed HC mice were housed two per cage with a perforated partition permanently separating the mice.

\section{Behavioral Analysis}

Social interaction test. All behavioral tests were performed as described previously (Lehmann and Herkenham, 2011). To determine the social interaction (SI) quotient, the mouse was placed in a $50 \times 50 \times 50 \mathrm{~cm}$ arena containing two perforated acrylic cylinders. One cylinder contained a CD-1 mouse and the other was empty. The mouse was left for 15 min under red lights while its position was recorded from above and subsequently automatically analyzed with TopScan (CleverSystems). The SI quotient was determined by dividing the time spent investigating the CD-1 mouse by the time spent investigating the empty cage. Lower scores were indicative of antisocial behavior.

Light/dark box test. The light/dark (L/D) test was conducted using a polycarbonate box $(50 \mathrm{~cm} \times 25 \mathrm{~cm}$ with $30 \mathrm{~cm}$ walls $)$ consisting of a dark compartment (one-third of the box) and a transparent compartment (two-thirds of the box) that was illuminated to $\sim 40$ lux. An open door divided the compartments. Each mouse was placed in the light compartment and allowed to freely move within the compartments for $10 \mathrm{~min}$. The time spent in the light compartment and the number of crosses between the light and dark sides were scored (SideScan, CleverSystems). Low scores were indices of anxiety-like behavior.

For the depletion repopulation experiments represented in Figures 4 and 5 , mice were run in two cohorts of six mice per group. The first cohort was exposed to the SI test only, and the second cohort was exposed to both behavioral tests then administered dihydroethidium (DHE) $1 \mathrm{~d}$ after the last test. In the first cohort, several mice were lost during the course of the 6 week experiment, which is reflected by the different $n$ values within this experiment.

\section{Immunohistochemistry}

Assessment of microglia depletion was performed by treating $C x 3 c r 1^{w t / g f p}$ mice with PLX5622 feed for $0,3,7$, or $14 \mathrm{~d}$ before they were killed. Mice were anesthetized with isoflurane and perfused transcardially with $0.9 \%$ saline followed by ice-cold phosphate-buffered $4 \%$ paraformaldehyde. Brains were removed and postfixed in the same fixative overnight followed by $25 \%$ sucrose in PBS for $24 \mathrm{~h}$. Coronal brain slices (30 $\mu \mathrm{m}$ thick) were collected on a freezing microtome. Free-floating sections were washed, blocked in $4 \%$ normal goat serum, incubated overnight at $4^{\circ} \mathrm{C}$ in monoclonal chicken anti-GFP (1: 5000; catalog \#ab13970, Abcam; RRID:AB_300798). Sections were rinsed and incubated for $1 \mathrm{~h}$ in secondary anti-chicken antisera labeled with Alexa Fluor-488 (1:1000; catalog \#A11039, Life Technologies). Sections were washed again with $0.1 \%$ Triton X-100 in $0.1 \mathrm{M}$ PBS (10 min, three times), counterstained with DAPI for $1 \mathrm{~min}$, washed with PBS, and coverslipped with polyvinyl alcohol (PVA)-DABCO (Sigma-Aldrich). Cells and sections were analyzed on a Zeiss 780 Confocal Microscope.

In studies examining ROS activity within microglia, free-floating sections from C57BL/6N mice were blocked, incubated overnight with rabbit anti-Iba1 (1:2500; catalog \#019-19741, Wako; RRID:AB_839504), washed, incubated in secondary antisera Alexa-Fluor 488 (1:1000; cata$\log$ \#A11034, Thermo Fisher Scientific), and counterstained with DAPI. 


\section{Reactive oxygen species measurement}

To measure oxidative stress in the form of ROS production, DHE (cata$\log$ \#D11347, Thermo Fisher Scientific), dissolved at $1 \mathrm{mg} / \mathrm{ml}$ in $100 \%$ DMSO then diluted 1:1 with sterile saline, was administered at $2.5 \mu \mathrm{g} / \mathrm{kg}$, i.p. Superoxide anions $\left(\mathrm{O}_{2}\right.$ radicals) are the primary form of ROS. DHE detects $\mathrm{O}_{2}$ radicals by a reaction between the two molecules that generates a membrane-impermeable, highly superoxide-specific, red fluorescent product 2-hydroxyethidium (2-OH-E), revealed with the proper excitation (Ex) and emission (Em) wavelengths (Ex/Em wavelength, 405: 600-650 nm; Zhao et al., 2005; Robinson et al., 2006; Zielonka et al., 2008; Nazarewicz et al., 2013). Importantly, commonly used 488-540 $\mathrm{nm}$ excitation wavelengths preferentially detect emissions from ethidium - produced by nonspecific oxidation reactions with DHEthat are thus not specific for superoxide (Nazarewicz et al., 2013; Cheng et al., 2018). Three hours after DHE administration, mice were perfused and processed as described above. Brains were sectioned at $30 \mu \mathrm{m}$ with a freezing microtome and counterstained with DAPI. Dried slidemounted sections were coverslipped with PVA-DABCO. Areas of interest were captured with a confocal microscope (model 780, Nikon), with a Zeiss $20 \times$ plan apochromat objective (0.8 aperture), and the files (.lsm) were analyzed for 2-OH-E fluorescence.

In each animal, four to six randomly selected fields in the infralimbic cortex (IL), prelimbic cortex (PL), nucleus accumbens (Acb), paraventricular nucleus of the hypothalamus (PVN), and dentate gyrus of the dorsal hippocampus (Hipp) were analyzed and averaged per mouse. The entire captured image was used for analysis. Ten $1 \mu \mathrm{m}$ maximum projection $z$-stacks (area $425 \times 425 \mu \mathrm{m}$ ) were reconstructed and analyzed using Volocity 6.3 (PerkinElmer). For separate analysis of ROS activity confined to microglia, the areas occupied by fluorescent staining of Ibal (ionized calcium binding adaptor molecule 1) antibody and 2-OH-E fluorescent signals were subjected separately in their respective channels to thresholding. First, a threshold 3 SDs higher than the median value was applied to the 2-OH-E signal, then 2-OH-E spots $<0.2 \mu \mathrm{m}^{2}$ diameter were excluded, and the remaining signal was measured. Second, microglia were thresholded $1 \mathrm{SD}$ higher than the mean, and objects between 100 and $1000 \mu \mathrm{m}^{2}$ were included. Next, 2-OH-E signal that completely overlapped with microglia signal was compartmentalized. After thresholding and compartmentalization, the integrated signal density for each thresholded image was calculated. Integrated density is defined as the product of pixel intensity $(255=$ the maximum pixel intensity for an 8 -bit image) $\times$ area $\left(\mu \mathrm{m}^{2}\right)$. Integrated densities of total 2-OH-E in the image and 2-OH-E compartmentalized within microglia were collected and further analyzed in Prism (GraphPad) and Excel (Microsoft).

\section{ROS blockade}

To block ROS production in the brain, NAC (catalog \#A7250, SigmaAldrich) was administered into the lateral ventricle via a chronically implanted cannula (Brain Infusion Kit \#3, Alzet). Administration was intracerebroventricular because NAC does not significantly cross the BBB (Sheffner et al., 1966; Bavarsad Shahripour et al., 2014). Under stereotaxic guidance in isoflurane-anesthetized mice, the tip of the cannula was placed $0.1 \mathrm{~mm}$ caudal to bregma, $1.0 \mathrm{~mm}$ lateral to the midline, and $2.4 \mathrm{~mm}$ below the skull. Correct placement was determined during processing for immunohistochemistry; only animals with tracks terminating in the ventricle were included in the study ( $>95 \%$ of all animals tested). The cannula was secured to the skull with cyanoacrylate adhesive (Loctite 454, Alzet). The cannula was connected via SILASTIC tubing to an osmotic minipump (\#2002, Alzet) implanted under the skin between the scapulae. The pump was loaded with NAC at $50 \mathrm{~mm}$ diluted in artificial CSF (aCSF) continuously delivered at the rate of $0.5 \mu \mathrm{l} / \mathrm{h}$ for 2 weeks.

\section{Statistical Analysis}

Behavioral data, 2-OH-E-integrated density, and microglial cell counts were summarized as the mean \pm SEM, and differences among experimental conditions were considered statistically significant when the $p$ value was $\leq 0.05$. Student's two-tailed $t$ tests, one-way ANOVA, and two-way repeated-measures ( $\mathrm{rm}$ ) ANOVAs, analyzed by SPSS (https:// www.ibm.com/analytics/us/en/technology/spss/) were applied where
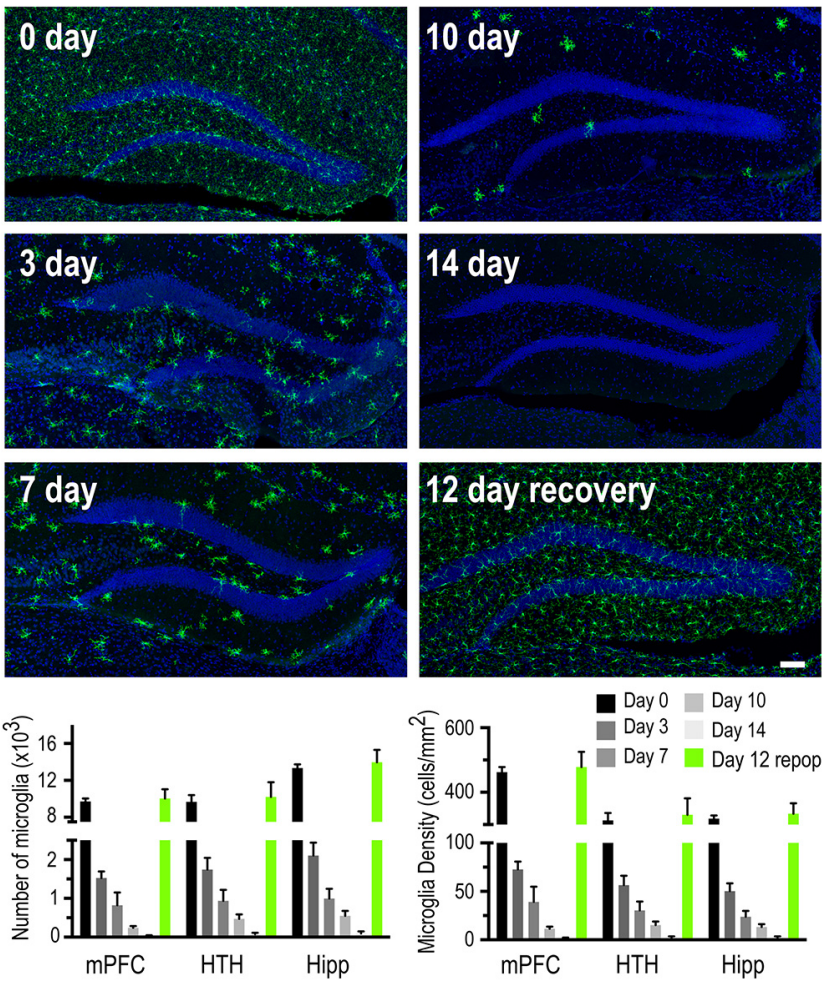

Figure 1. PLX5622 in the chow depleted microglia over a 2 week period, and microglia rapidly returned after the drug was discontinued. Images at the top show examples of microglia visible in the hippocampus of CX3CR1-GFP mice at the times indicated. Graphs show quantification of cell counts in Hipp, whole hypothalamus (HTH), and mPFC. Scale bar, $100 \mu \mathrm{m}$. repop, Repopulation.

appropriate to assess between-subject comparisons along the variables behavioral condition versus, for example, behavioral or biological assay score. Post hoc tests were performed with Bonferroni's procedure and Sidak's multiple-comparison test, and the $p$ level was adjusted for multiple comparisons.

\section{Results}

Microglia depletion protects against behavioral abnormalities in CSD-stressed mice

We first verified that continuous PLX5622 administration in the chow almost completely depleted microglia, and that, upon discontinuation of the drug, microglia rapidly and homeostatically repopulated the brain (Elmore et al., 2014; Dagher et al., 2015). CX3cr1 $1^{w t / g f p}$ mice were fed PLX5622 for $0,3,7,10$, and $14 \mathrm{~d}$. Microglia numbers, assessed by the visualization of GFP-labeled microglia, were decreased by $81 \%$ after $3 \mathrm{~d}$ and by $>99 \%$ after $14 \mathrm{~d}$ of treatment, and they were fully recovered to normal levels at $12 \mathrm{~d}$ after drug discontinuation (Fig. 1).

We next determined that the depletion per se did not affect behavior. Anxiety-like and social behaviors were measured in unstressed (HC) control and PLX5622-treated mice at 2 and 4 weeks after drug initiation (Fig. 2a). L/D crossings (Fig. 2b) and SI ratios (Fig. $2 c$ ) showed no significant main effect of depletion (two-way repeated-measures ANOVA, drug effect: $\mathrm{L} / \mathrm{D}, F_{(1,10)}=$ $0.46, p=0.5$; SI, $\left.F_{(1,10)}=1.2, p=0.3\right)$. Thus, we concluded that CSF1R inhibition allows for effective microglia depletion without dramatically affecting behavioral measures of anxiety and social behavior. Similar findings have been obtained with other behavioral tests of motor function, anxiety, and spatial learning (Elmore et al., 2015). 
a

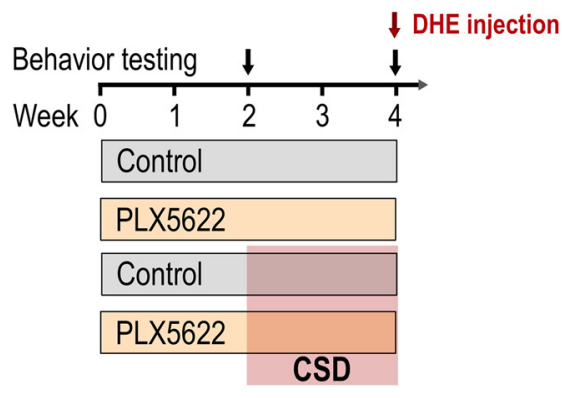

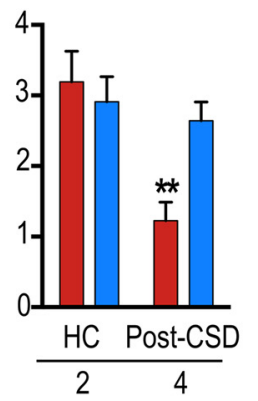

b
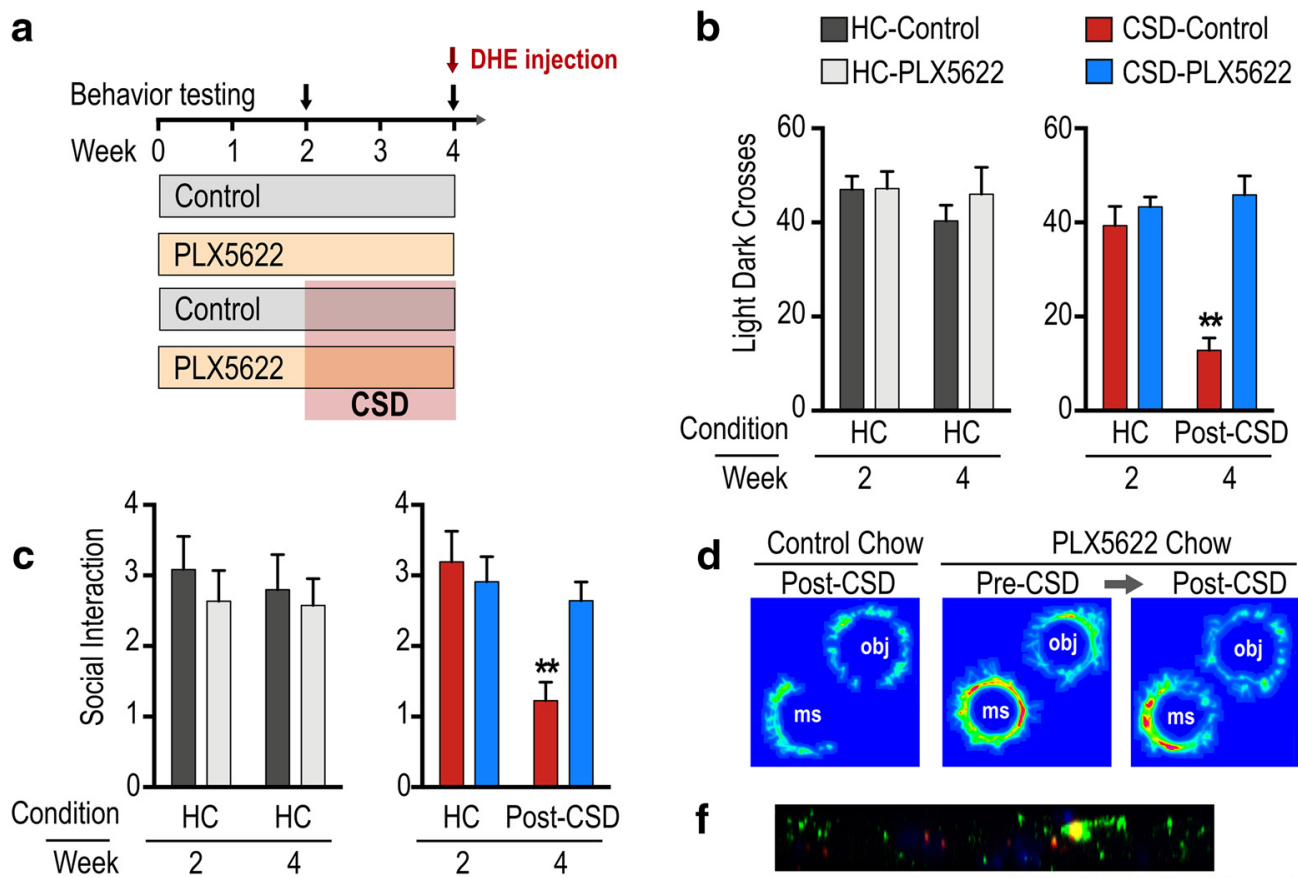

d

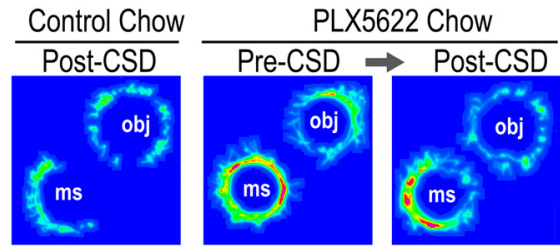

e
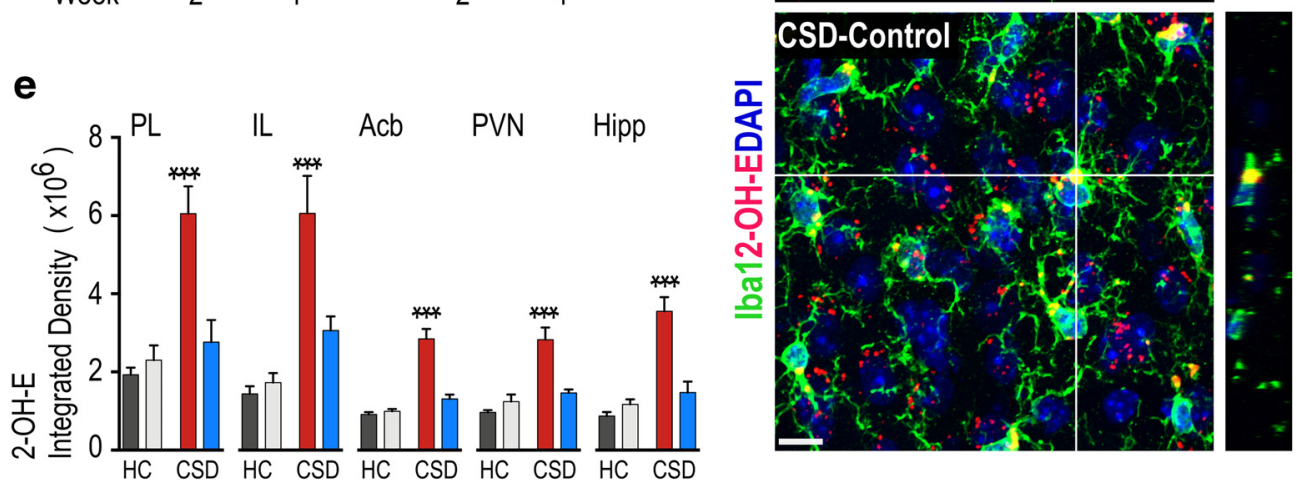

g

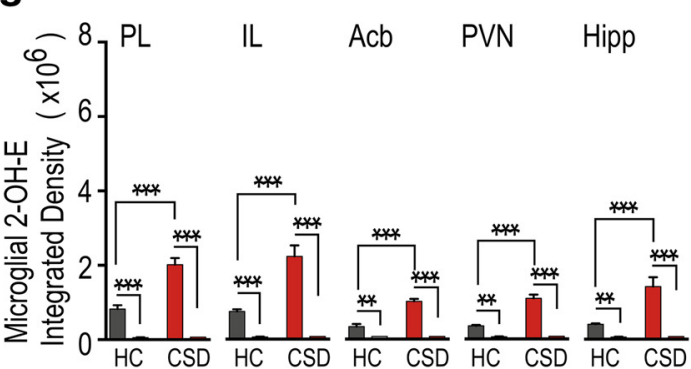

h CSD-PLX5622

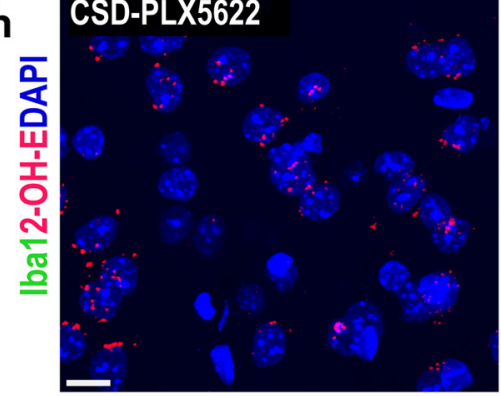

Figure 2. Microglial depletion with PLX5622 protected mice from the adverse effect of CSD and blocked the elevation in ROS activity in all selected brain areas. $\boldsymbol{a}$, The experimental design. $\boldsymbol{b}$, Light dark (L/D) scores show reduced crosses to light in the CSD control group only. $c$, Social interaction (SI) scores show reduced SI in the CSD control group only. $\boldsymbol{d}$, Representative heat maps of behavior for mice in two groups in the SI task; one control mouse after CSD and one depleted mouse tested before and after CSD. $\boldsymbol{e}, \boldsymbol{g}, \mathrm{ROS}$ activity assayed by 2-OH-E fluorescence integrated density in all cells $(\boldsymbol{e})$ and in microglia $(\boldsymbol{g}) . \boldsymbol{f}, \boldsymbol{h}$, Confocal image showing colocalization of 2-0H-E in Iba1-positive cells of a CSD-control mouse $(\boldsymbol{f})$ contrasted with reduced 2-OH-E labeling and no detectable Iba1 immunostaining in a CSD-PLX5622 mouse (h). Images were taken from prefrontal cortex. In unstressed HC mice, microglial depletion did not alter affective behavior or ROS activity in selected brain areas (data in text). $N=6$ per group. ${ }^{* *} p<0.01,{ }^{* * *} p<0.001$. ms, Mouse; obj, object. Scale bars, $10 \mu \mathrm{m}$. Time on social target and object from the SI task are shown in Figure 2-1, available at https://doi.org/10.1523/JNEUROSCI.0184-19.2019.f2-1.

We then examined the effects of microglia depletion on stress effects. Microglia-depleted and nondepleted mice were behaviorally phenotyped before and after CSD (Fig. 2a). Depletion did alter the behavioral response to CSD in the $\mathrm{L} / \mathrm{D}$ and SI tests: $\mathrm{L} / \mathrm{D}$ crosses (two-way rm ANOVA, $F_{(1,1)}$; interaction effect: $F=27.7$, $p=0.0004$; time effect: $F=18.9, p=0.001$; drug effect: $F=23.8$, $p=0.0006$; Fig. $2 b$ ); and SI ratios (two-way rm ANOVA, $F_{(1,10)}$; interaction effect: $F=6.1, p=0.03$; time effect: $F=10.4, p=$
0.01; but no main effect of drug, $p=0.11$; Fig. $2 b, c)$. Post hoc analyses showed a protective effect of microglia depletion in CSD. In the L/D test, anxiety-like behaviors induced by CSD were not observed in microglia-depleted mice (Sidak's multiple-comparison test, $p=0.0001$ ). Post hoc analysis also showed that mice fed control chow displayed pronounced social avoidance after social defeat, whereas microglia-depleted mice showed social behaviors comparable to prestress conditions (Sidak's multiple-comparison test, 
$p=0.009$ ) (Fig. $2 c$ and Fig. 2-1, available at https://doi.org/10.1523/ JNEUROSCI.0184-19.2019.f2-1). Heat maps of investigative behavior during the SI task highlight the behavioral differences between depleted and nondepleted mice (Fig. $2 d$ ). Thus, the behavioral resiliency observed in microglia-depleted mice implies that microglia activation during CSD renders mice susceptible to the adverse effects of CSD.

Parenthetically, most microglia-intact mice that undergo the CSD paradigm subsequently show elevated anxiety and antisocial behaviors, but a small percentage $(<20 \%)$ are resilient to the CSD effects and do not show behavioral declines (Lehmann et al., 2018). We are not able in the depletion study to predict beforehand which mice might be susceptible versus resilient to the CSD, so we cannot say whether some depleted mice that showed resilience might have done so without depletion. Similarly, in all experiments in this study, we do not address the susceptibilityresilient spectrum of behaviors naturally found in our mice populations.

\section{Microglia depletion abolishes elevated ROS response to CSD}

We examined the interaction between CSD and microglial depletion on ROS activity in several cortical and subcortical areas (Fig. $2 e-h)$. 2-OH-E fluorescence labeling of ROS activity was performed $24 \mathrm{~h}$ after the last behavioral observation and was quantified with confocal microscopy. Two-way ANOVA comparing the effects of microglia depletion and stress revealed significant interactions on 2-OH-E fluorescence in each examined region: two-way ANOVA, $F_{(1,20)}$; PL: $F=13.6, p=0.001$; IL: $F=9.4, p=$ 0.006; Acb: $F=31.39, p=0.0001$; PVN: $F=19.4, p=0.0003$; and Hipp: $F=24.4, p=0.0001$. Bonferroni post hoc analysis revealed similar patterns of significant changes across regions; nondepleted CSD mice showed substantial 2-OH-E production activity compared with all other conditions. Importantly microglia depletion reduced the effects of stress on ROS activity to levels that did not differ from those in unstressed mice (Fig. 2e).

There were regional differences between levels of activity (integrated density $\times 10^{6}$ ). Within each condition, higher levels of activity were seen in the PL and IL medial prefrontal cortices compared with the Acb, PVN, and Hipp. For example, in nonstressed samples, prefrontal cortical regions had two units of activity compared with other regions, which all had similar levels $(\sim 1$ unit).

Microglial ROS production compared with production in all cells was lower overall, but with the same pattern of relatively elevated activity in the prefrontal cortical areas versus other areas (Fig. 2g). Microglial 2-OH-E production from CSD mice was elevated compared with nonstressed mice in the nondepleted groups, and it was absent in the depleted groups. Two-way ANOVA indicated significant interaction effects on 2-OH-E fluorescence in each examined region (two-way ANOVA, $F_{(1,20)}$; PL: $F=33.9, p=0.0001 ;$ IL: $F=21.2, p=0.0002$; Acb: $F=48.6, p=$ 0.0001; PVN: $F=54.6, p=0.0001$; Hipp: $F=14.4, p=0.001$; Fig. $2 g$ ). Comparing the levels of 2-OH-E production in Figure 2, $e$ and $g$, it is noteworthy that the contribution of microglial ROS to total ROS elevated by CSD is $\sim 25 \%$. Given that microglia comprise only 10-15\% of all cells in brain (Lawson et al., 1990), the microglial ROS contribution is significant.

\section{ROS blockade during CSD protects against behavioral abnormalities caused by stress}

The striking effect of microglia depletion on affective behavior made us ask whether this was attributable to elevated ROS activity during stress. To examine this, mice were administered the anti- oxidant NAC or vehicle (aCSF) delivered continuously intracerebroventricularly during the CSD procedure or in nonstressed $\mathrm{HC}$ control mice (Fig. $3 a$ ). NAC prevented the CSD-induced drop in social interaction in the SI task and had no effect in HC mice (two-way ANOVA interaction effect between drug and stress: $F_{(1,16)}=8.1, p=0.01$; Fig. $3 b, c$ and Fig. $3-1$, available at https://doi.org/10.1523/JNEUROSCI.0184-19.2019.f3-1).

NAC treatment completely blocked the CSD-induced rise in 2-OH-E production both in all cells (Fig. $3 d$ ) and in microglia (Fig. 3e). It had no effect on basal levels of $2-\mathrm{OH}-\mathrm{E}$ production in both cell populations. Two-way ANOVA detected significant drug $\times$ stress interactions on 2-OH-E fluorescence in each examined region: interaction effect two-way ANOVA, $F_{(1,16)}$; PL: $F=$ $17.2 ; p=0.0005$; IL: $F=22.2, p=0.0002$; Acb: $F=6.9, p=0.02$; PVN: $F=15.8, p=0.0009$; Hipp: $F=7.1, p=0.02$. Post hoc analysis showed that ROS activity was significantly elevated in vehicle-treated CSD mice compared with all other groups, and this effect was completely blocked in NAC-treated CSD mice $(p<0.01$ for each region; Fig. $3 d)$. Drug and stress interactions on 2-OH-E fluorescence were also extended to microglia populations in all regions (interaction effect, two-way $\operatorname{ANOVA,~} F_{(1,16)}$; PL: $F=5.5, p=0.03$; IL: $F=12.7, p=0.002$; Acb: $F=8.4, p=$ 0.009; PVN: $F=5.6, p=0.03$; Hipp: $F=16.1, p=0.0007)$. Microglial 2-OH-E fluorescence was significantly elevated in vehicle-treated CSD mice compared with all other groups (Fig. $3 e$ ). This effect was observed in all areas except the prelimbic cortex; here post hoc tests detected significant changes only between vehicletreated unstressed mice and CSD mice $(p<0.005)$.

\section{Microglia repopulation after CSD removes protection against stress-induced behavioral changes and drives ROS activity visualized by $2-\mathrm{OH}-\mathrm{E}$ production}

The effect of depletion and repopulation on behavior and 2-OH-E production was first measured in unstressed mice (Fig. $4 a)$. Two-way ANOVA detected no significant behavioral effects of depletion or repopulation (L/D time effect: $F_{(3,30)}=2.1, p=$ 0.18 , Fig. $4 b$; SI time effect: $F_{(3,66)}=2.4, p=0.1$, Fig. $4 c, d$ and Fig. 4-1, available at https://doi.org/10.1523/JNEUROSCI.018419.2019.f4-1). ROS activity, assessed at the end of the experiment, was not significantly affected by treatment. Unstressed mice that underwent depletion and repopulation compared with untreated control mice showed a trend for greater 2-OH-E intensities in the $\mathrm{PL}(t=2.4, p=0.04), \mathrm{IL}(t=3.0, p=0.013)$, and PVN $(t=3.5$, $p=0.005)$, but no significance was detected in data corrected for multiple comparisons (Bonferroni cutoff, $p<0.001$ ). 2-OH-E intensities in the Acb $(t=1.8)$ and HIPP $(t=0.9)$ were not different between groups ( $p>0.05$; Fig. 4e). Microglial 2-OH-E intensity approached significance in the infralimbic cortex $(t=$ 3.6, $p=0.004$; Bonferroni cutoff, $p<0.001$; Fig. $4 g$ ). All other examined regions showed no effect of drug regimen on microglial 2 -OH-E intensity $(t=0.7,0.4,1.9$, and 0.3 , respectively, in the PL, Acb, PVN, and HIPP; $p>0.05)$. Confocal images from IL cortex further demonstrate the minimal effect of depletion/repopulation in nonstressed mice (Fig. $4 f, h$ ). Thus, microglial depletion and repopulation by itself leaves no imprint on behavior and ROS activity assessed by $2-\mathrm{OH}-\mathrm{E}$ production.

We next asked whether microglial repopulation would influence behavior in previously stressed mice; thus, behavioral trajectories in all groups were monitored before and during the repopulation period (Fig. 5a). Microglia depletion and repopulation had biphasic effects on behavioral response to stress in the $\mathrm{L} / \mathrm{D}$ and SI tests [L/D crosses (two-way rm ANOVA; time main effect: $F_{(3,45)}=15.45, p=0.0001$; group main effect: $F_{(2,15)}=$ 
a
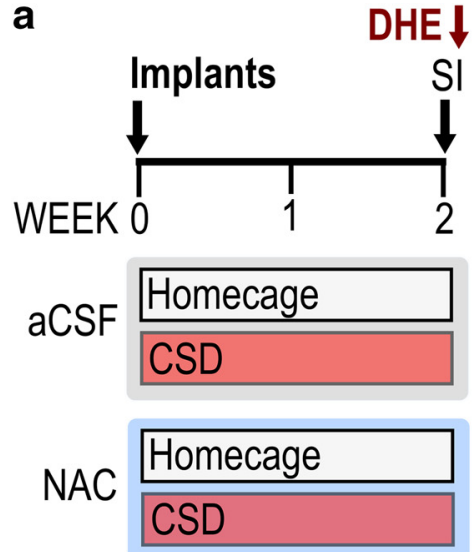

b

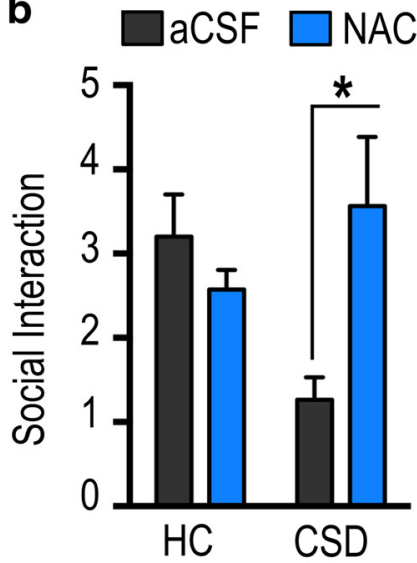

c CSD+aCSF
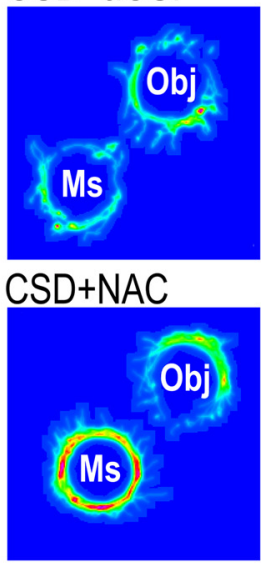

Hipp

d

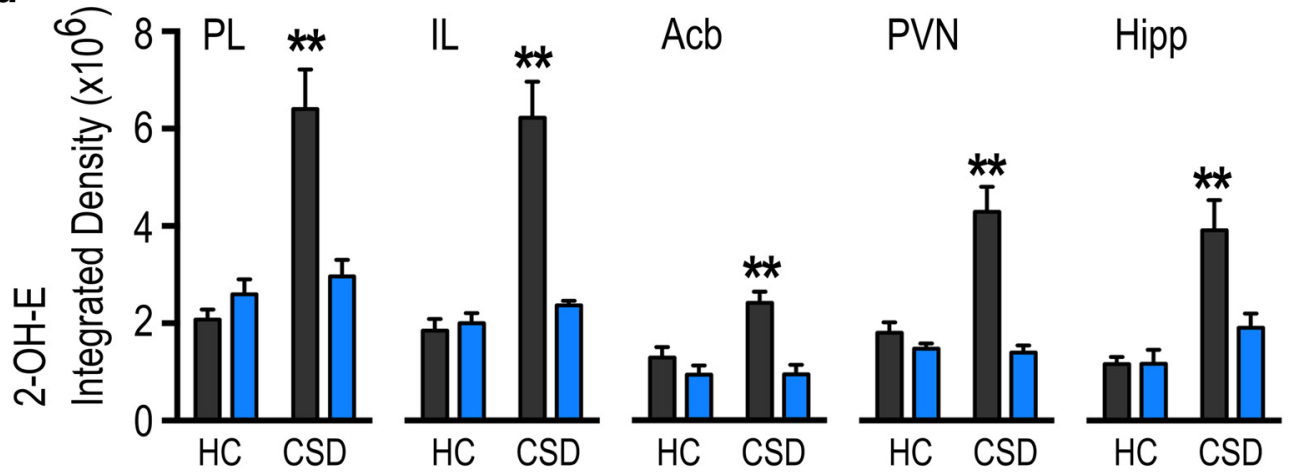

e

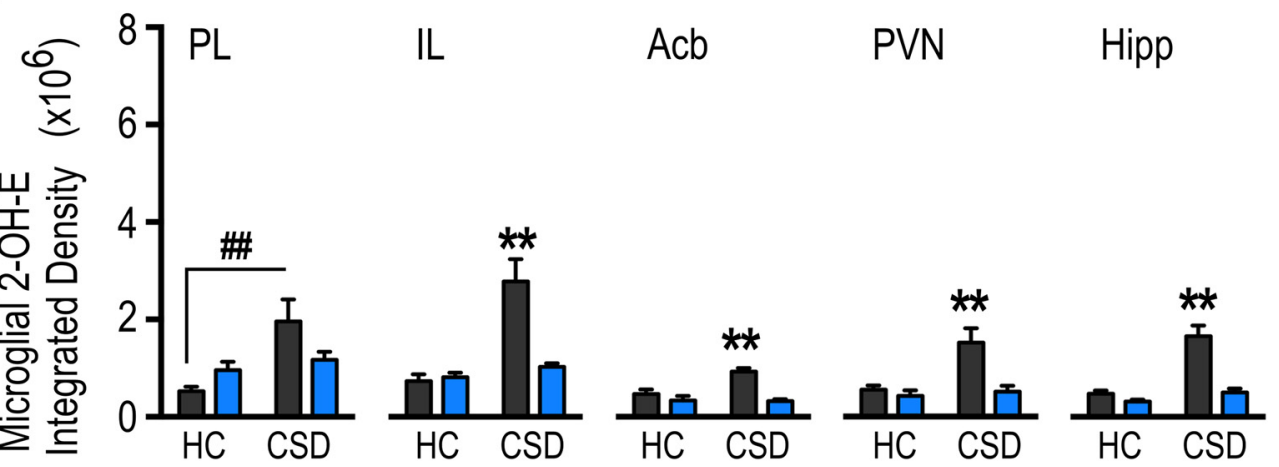

Figure 3. Intracerebroventricular NAC administration during CSD prevented stress-induced behavioral decline, and it reduced ROS activity, measured by 2-OH-E fluorescence, in all measured brain areas. $\boldsymbol{a}$, Experimental design. $\boldsymbol{b}$, Social interaction scores in NAC-treated CSD mice were elevated (to unstressed levels) compared with aCSF-treated CSD mice (N $=5 /$ group). $\boldsymbol{c}$, Heat maps show a decline in preference for social targets after CSD only in the vehicle-treated mouse. $\boldsymbol{d}, \boldsymbol{e}$, Total ROS activity $(\boldsymbol{d})$ and microglial ROS activity $(\boldsymbol{e})$ were elevated only in untreated CSD mice. ${ }^{*} p<$ $0.05,{ }^{* *} p<0.01$, \#\#p $<0.01$. ms, Mouse; obj, object. Time spent investigating social target and object from the SI task are shown in Figure 3-1, available at https://doi.org/10.1523/JNEUROSCI. 0184-19.2019.f3-1.

13.55, $p=0.0005$; interaction: $F_{(6,45)}=4.65, p=0.001$; Fig. $\left.5 b\right)$ and SI ratios (time: $F_{(3,84)}=13.44, p=0.0001$; group: $F_{(2,28)}=$ $17.52, p=0.0001$; interaction: $F_{(6,84)}=7.45, p=0.001$; Fig. $5 c, d$ and Fig. 5-1, available at https://doi.org/10.1523/JNEUROSCI. 0184-19.2019.f5-1)]. Highly significant interaction effects allowed us to rerun ANOVA with simple effects. The data showed a protective effect of microglia depletion-whereas CSD produced anxiety-like and antisocial behaviors in control mice, PLX5622treated stressed mice showed behaviors similar to unstressed mice ( 4 week post-CSD observation: L/D crosses: $F_{(2,15)}=17.57$, $p=0.0001$; SI: $F_{(2,28)}=15.2, p=0.0001$; Fig. 5b,c). Thus, the protective effect of depletion seen earlier (Fig. 2) was replicated. Surprisingly, when the same mice were tested again after micro- glial repopulation following drug discontinuation, the deleterious effect of CSD now emerged for both $\mathrm{L} / \mathrm{D}$ crosses (Fig. $5 b$ ) and SI ratios (L/D crosses: $F_{(3,15)}=7.6, p=0.01 ;$ SI: $F_{(3,24)}=34.1, p=$ 0.0001 ; Fig. $5 c, d)$. Stressed mice with no drug treatment continued to show reduced scores at 2 weeks after CSD, and the continuously depleted mice continued to be protected from the deleterious CSD stress effects (L/D crosses: $F_{(2,15)}=13.8, p=$ 0.0004; SI: $\left.F_{(2,28)}=11.9, p=0.0002\right)$.

The behavioral observations at the end of this experiment had parallels in 2-OH-E intensity in brain. CSD had a lasting elevation of 2-OH-E production only in mice with microglia present during the 2-week recovery phase post-CSD (Fig. $5 a$, control and repopulation conditions). Both groups showed no relative differ- 
ence in 2-OH-E fluorescence. In mice with continued depletion, 2-OH-E intensities were dramatically reduced in all cells (Fig. $5 e, h$; one-way ANOVA, $F_{(2,17)}$; PL: $F=27.2, p=0.0001 ;$ IL: $F=13.8, p=$ 0.0004; Acb: $F=11.4, p=0.001$; PVN: $F=9.03, p=0.003$; HIPP: $F=11.4, p=$ $0.001)$. Similar effects were observed in microglial 2-OH-E fluorescence intensities; both control and repopulated brains had substantial and similar microglial 2-OH-E levels compared with continuously depleted brains (Fig. $5 f-h$; one-way ANOVA $F_{(2,17)}$; PL: $F=47.7, p=0.0001$; IL: $F=19.8, p=0.0001$; Acb: $F=8.5, p=$ 0.003; PVN: $F=25.9, p=0.0001$; Hipp: $F=16.1, p=0.0002)$. Notably, repopulating microglia appear to be necessary for elevated ROS activity in other cells in the recovery period.

\section{ROS blockade post-CSD protects}

against the adverse behavioral effects of microglial repopulation

The results thus far show that microglial depletion during CSD prevented adverse affective changes and blunted ROS activity, but microglia repopulation post-CSD unmasked behavioral declines and elevated ROS activity. Additionally, buffering ROS activity by NAC administration during CSD protected against adverse behavioral changes. We were interested to learn whether buffering ROS activity during repopulation would have a similar protective effect. To examine this, NAC was administered intracerebroventricularly in the post-CSD repopulation period in a $2 \times 2$ experimental design that explored the interaction between microglial repopulation and ROS activity (Fig. 6a). During CSD, mice received either PLX5622 feed or normal chow, then normal chow upon the cessation of CSD. During the recovery period, mice received either NAC or aCSF intracerebroventricularly. Thus, the four groups were (1) control stressed mice, (2) mice that were treated with PLX5622 through the CSD stress period and then removed from receiving drug for 2 weeks, (3) control mice that received NAC in the poststress repopulation period, and (4) PLX5622-treated mice that received NAC in the repopulation period (Fig. $6 a$ ). Mice were behaviorally phenotyped three times, as follows: (1) before the CSD period, (2) at the end of the stress period, and (3) at the end of the repopulation period. At the end of behavioral observations, mice were assayed for 2-OH-E production. Two-way ANOVA showed a significant main effect of time $\left(F_{(2,40)}=21.46, p=0.0002\right)$ but not of treatment $\left(F_{(3,20)}=\right.$ $0.6, p=0.623)$ and a significant interaction effect $\left(F_{(6,40)}=8.94\right.$, $p<0.0001)$ in $\mathrm{L} / \mathrm{D}$ crosses, and a significant main effect for time $\left(F_{(2,40)}=10.99, p<0.005\right)$ but not treatment $\left(F_{(3,20)}=2.83, p=\right.$ $0.74)$ and a significant interaction $\left(F_{(6,40)}=10.52, p<0.0001\right)$ for SI ratios. Control mice showed reduced $\mathrm{L} / \mathrm{D}$ crosses and SI ratios at both time points poststress ( $p<0.01$ for all; Fig. $6 b-d$ and Fig. 6-1, available at https://doi.org/10.1523/JNEUROSCI.0184-19.2019.f61). Control mice that received NAC in the poststress period showed behavioral recovery. In contrast, as before (Fig. 2), PLX5622-treated mice were protected from CSD effects immediately poststress and showed reduced scores relative to prestress scores after 2 weeks of recovery in both tasks $(p<0.01)$. Importantly, the PLX5622-treated group that received NAC in the recovery period did not show the same decline in scores, indicating that the blockade of ROS in the poststress period protected against the deleterious effect on the behavior of microglial repopulation after stress.

ROS activity was assessed in the four groups at the end of the repopulation (Fig. 7). CSD-stressed mice showed levels of 2-OH-E production that were not different between untreated and microglia-depleted mice measured after repopulation, and both groups showed ROS levels greatly reduced by intracerebroventricular NAC treatment (implant effect: $F_{(1,20)}=9.1-85.1$ for the regions; $p<0.01$ to $p<0.0001$; Fig. $7 a, c)$. Levels of $2-\mathrm{OH}-\mathrm{E}$ fluorescence were approximately twice as high in $\mathrm{mPFC}$ regions compared with other areas, as they had been just after CSD (Fig. 2). Within repopulated microglia, 2-OH-E levels were likewise high (relative to never-stressed mice) in both control and repopulated brains, and NAC reduced 2-OH-E fluorescence in both (implant effect: $F_{(1,20)}=10.8-120.5$ for the regions; $p<0.01$ to $p<0.0001$; Fig. $7 b, d)$. 

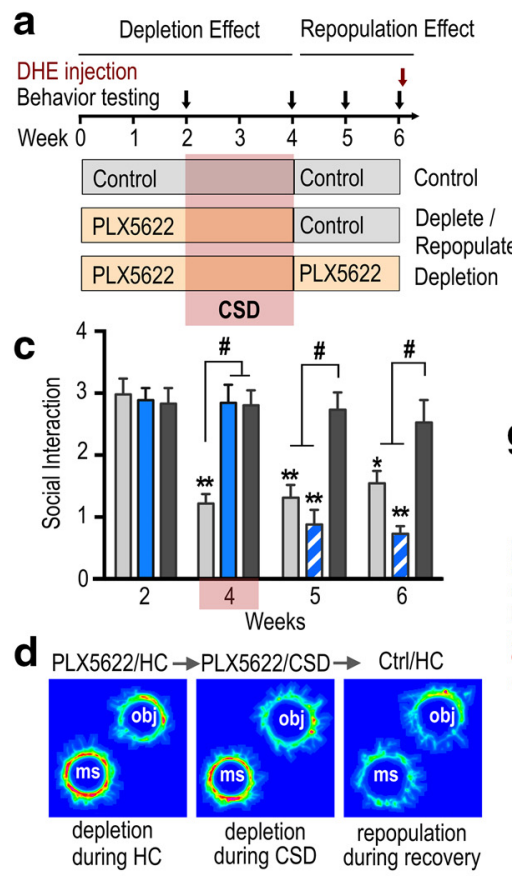

e
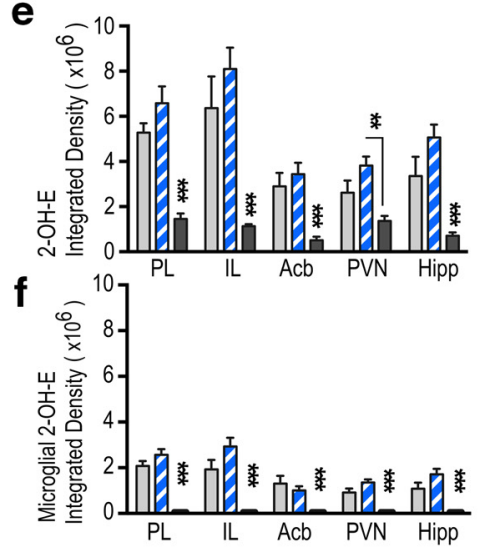

\section{西}
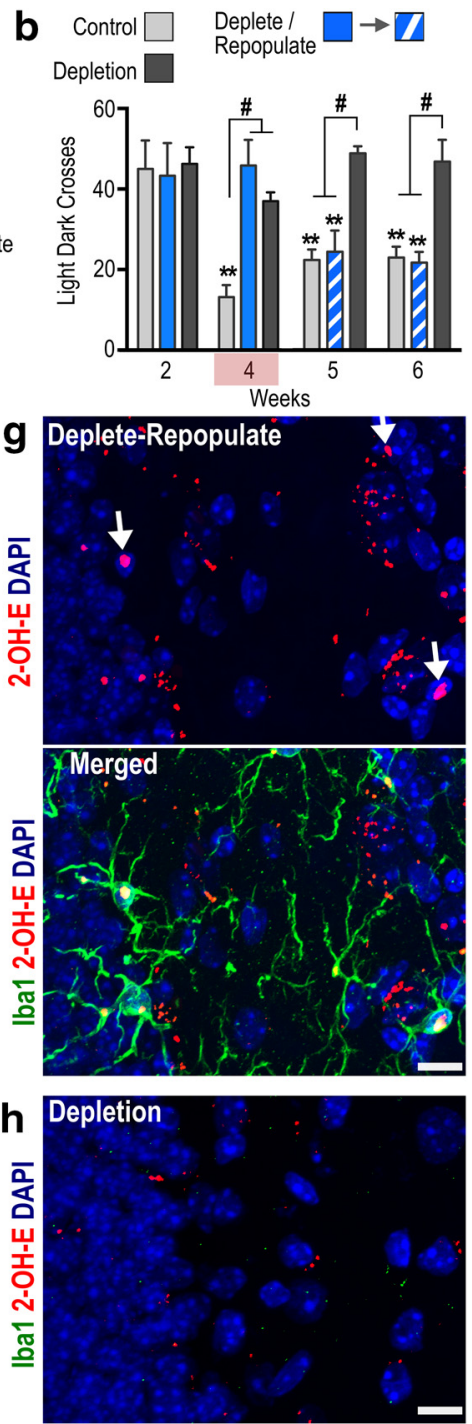

Figure 5. Microglial depletion protected against behavioral effects of stress and reduced ROS activity in widespread brain areas (assayed at 6 weeks); upon repopulation, behavioral protection was lost, and ROS activity rebounded. $\boldsymbol{a}$, The experimental paradigm. $\boldsymbol{b}$ - $\boldsymbol{d}$, Mice with intact microglia during CSD (control) showed reduced L/D crosses $(\boldsymbol{b})$ and SI ratios ( $\boldsymbol{c}, \boldsymbol{d})$ measured after CSD, this effect persisted for 2 weeks after the cessation of stress. Mice without microglia (depletion) showed poststress behavioral responses that were comparable to prestress observations. Sharp behavioral declines were observed after microglia recovered (deplete/repopulate). $\boldsymbol{d}$, Heatmap showing the behavioral trajectory of a single mouse that experienced depletion during $\mathrm{HC}$ and $C S D$, then repopulation during recovery. $N=6 /$ group for $L / D$ and $N=9$ for depletion/repopulation group; 10 for depletion group, and 12 for control group for the SI task. Significant Bonferroni post hoc comparisons were detected. ${ }^{* *} p<0.01$ or ${ }^{*} p<0.05$ vs within-group nonstressed measurement acquired at 2 weeks; $\# p<0.05$ vs other groups within a time point bin. $\boldsymbol{e}-\boldsymbol{h}$, Confocal microphotographs and quantitative analysis show experimental effects on 2-OH-E fluorescence. $\boldsymbol{e}, \boldsymbol{f}$, Microglia depletion significantly altered 2-OH-E fluorescence in numerous brain regions, measured in all cells $(\boldsymbol{e})$ and in microglia $(\boldsymbol{f})$. $\boldsymbol{g}$, Confocal microphotographs of the dentate hilus and granule cell layer show 2-OH-E fluorescence and Iba 1 expression in the post-CSD recovery group top, 2-OH-E and DAPI; bottom, merged with Iba1 staining. Arrows mark large spots that appear only in microglia. $\boldsymbol{h}$, Confoca image shows reduced 2-OH-E labeling and no Iba1 expression in the depletion group. $\mathrm{N}=6 /$ group. ${ }^{* *} p<0.01,{ }^{* * *} p<0.001$. Scale bar, $10 \mu \mathrm{m}$. Time on social target and object from the SI task are shown in Figure 5-1, available at https://doi. org/10.1523/JNEUROSCI.0184-19.2019.f5-1.

\section{Discussion}

We examined the roles of microglia and oxidative stress in anxiety-like states induced by the psychosocial stressor CSD. Microglial depletion, by administration of the CSF1R antagonist PLX5622 in the chow, by itself did not alter affective behavior, but depletion during the CSD period prevented the development of CSD-induced anxiety-like and antisocial behaviors. Continued depletion during the 2 week poststress period was associated with continued protection against deleterious stress effects. Surprisingly, when drug was discontinued after the CSD sessions ended and microglia had repopulated the brain, the repopulated animals now showed deficits in the behavioral assays, as did animals that had not been depleted. ROS activity (measured by 2-OH-E fluorescence) strongly mirrored declines in affective behavior; ROS activity was elevated by CSD and remained elevated in the poststress periods. Microglial depletion either during CSD or during CSD and recovery prevented ROS elevations in response to stress. However, if microglia repopulated brains of previously stressed mice, then robust ROS production was also detected. We showed a causal relation between ROS activity and anxiety-related behaviors; blockade of ROS activity by intracerebroventricular administration of the antioxidant NAC during CSD prevented the stress-induced behavioral declines, and ROS blockade during the repopulation period reversed the behavioral declines in both intact and repopulated stressed mice. The data suggest that microglia present during CSD drive ROS production, and this activity underlies the adverse behavioral effects of CSD. The findings are summarized in Figure 8.

The present findings merge two separate lines of inquiry about mechanisms by which chronic psychosocial stress alters emotional behavior. First, we showed the importance of microglia in mediating the effect of CSD. Previous studies have shown that stressors like CSD alter microglial activity (Tynan et al., 2010; Wohleb et al., 2014; Lehmann et al., 2016), suggesting that microglia are involved in CNS responses to stress, and now through a depletion strategy, we showed that microglia play a necessary role in that action, supporting recent work on repeated social defeat-induced anxiety (McKim et al., 2018). Second, previous studies have demonstrated a role for oxidative stress in mediating the effects of stress on affective behavior (Schiavone et al., 2009; Seo et al., 2012; Ibi et al., 2017). Now, by examining these aspects concurrently, we offer new insights into the causal mechanisms played by microglia.

Previous studies have demonstrated the importance of CNS ROS in influencing behavior, but much of that work emphasized the dysregulation of ROS production in neurons. In one study in anxious versus nonanxious mice, anxiety was correlated with ROS activity in neurons and glia (Rammal et al., 2008). In other studies, stress-induced depressive-like states were shown to be ROS dependent (Schiavone et al., 2009; Seo et al., 2012; Ibi et al., 2017), with histological evi- 
a

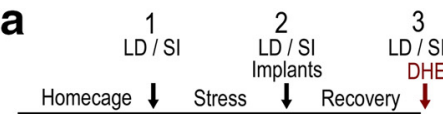

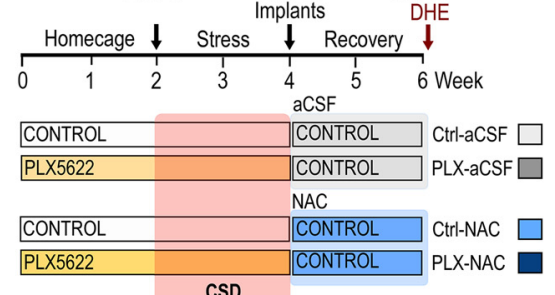

b

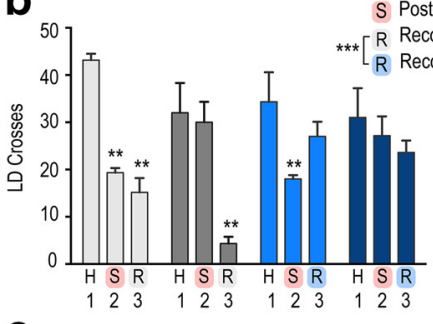

C

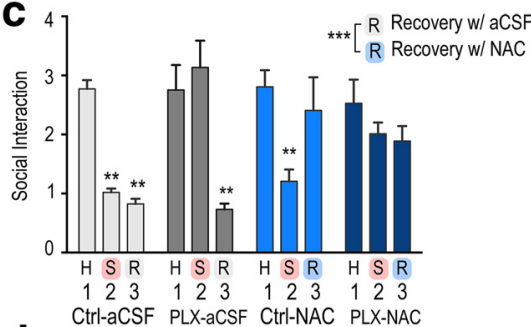

d

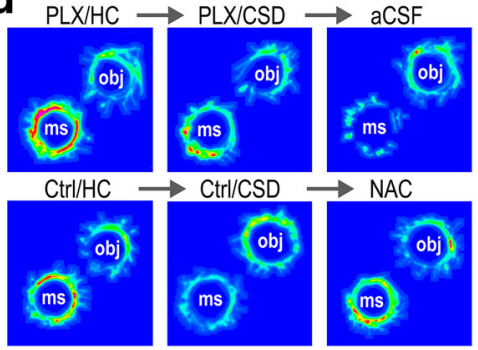

Figure 6. ROS blockade with intracerebroventricular NAC during microglial recovery prevented the belated deleterious stress effect on behavior seen during repopulation. It also permitted behavioral recovery in previously CSD-stressed mice that did not have microglial depletion. $\boldsymbol{a}$, The behavioral paradigm with the three time points of behavioral measurements marked. $\boldsymbol{b}, \mathrm{L} / \mathrm{D}$ crosses. $\boldsymbol{c}$, SI ratios. $\boldsymbol{d}$, Representative heatmaps of behavior for mice in two groups in the SI task; one mouse with depleted microglia before and after CSD (representing the PLX-a(SF group), and then during recovery with repopulation; and one intact mouse tested before and after CSD, and during recovery while treated with NAC (representing the Ctrl-NAC group) ${ }^{* *} p<0.01,{ }^{* * *} p<0.001 . N=5 /$ group. Time on social target and object from the SI task are shown in Figure 6-1, available at https://doi.org/10.1523/JNEUROSCI.018419.2019.f6-1.

dence for neuronal ROS induction in each study. Notably, these studies used DHE to localize ROS activity, but they used fluorescent excitation with longer wavelengths that predominantly detect fluorescent emissions from ethidium (E), which is generated by additional oxidation events other than superoxide reactions with DHE generating 2-OH-E (Nazarewicz et al., 2013; Cheng et al., 2018).

We found that CSD elicited superoxide-mediated ROS production in both microglia and nonmicroglial cells. Interestingly, without microglia, ROS activity in all other cells was greatly reduced (Fig. $2 e$, depletion group), suggesting that microglia and microglial ROS activity drive oxidative stress pathways in other cells, possibly neurons, after CSD.
Mitochondria are the major source of superoxide production in the cell (Turrens, 2003). High-magnification analysis of confocal images indicated that the 2-OH-E oxidation products of DHE were discretely localized as small spots in the cytoplasm. These may be associated with superoxide radicals formed in the electron transport chain in mitochondria (Forkink et al., 2010; Finkel, 2012; Rojo et al., 2014), a hypothesized target of psychological stress (Picard and McEwen, 2018). In addition, phagosomal NADPH oxidase activity generates ROS in phagosomes (Lambeth, 2004; Minakami and Sumimotoa, 2006; Quinn et al., 2006). Larger spots, which appeared only in microglia (Fig. $5 g$, arrows), may mark ROS production in phagosomes, as we previously noted (Lehmann et al., 2018).

Our data show the importance of ROS activity and microglia in controlling mood states. Multiple mechanisms contribute to oxidative stress in the brain (Cobley et al., 2018). Various forms of psychophysiological stress have been reported to increase multiple markers of oxidative stress (for review, see Seo et al., 2012; Schiavone et al., 2013; Ibi et al., 2017). Elevations in glucocorticoids have been considered as a possible mechanism for stress effects on ROS activity (Zafir and Banu, 2009). An older view holds that glucocorticoids can "endanger" neurons via oxidative stress (Sapolsky, 1999). More recently, the role of glucocorticoids in influencing CNS neuroinflammation has been explored (Sorrells et al., 2009; Frank et al., 2015), and less emphasis has been placed on oxidative stress. Oxidative stress and inflammation can positively interact with each other, such that cause and effect are difficult to assign when feedforward cascades lead to loss of homeostasis.

It is perplexing that repopulated microglia, which did not exist in the brain at the time of the CSD sessions, appeared to exert or contribute to a proanxiety and antisocial state in the microgliarepopulated mice that had been previously protected from the stress effects. Repopulation occurs entirely from surviving residual microglia in the brain (Huang et al., 2018; Weber et al., 2019). Newly proliferated microglia following a stressful event did not themselves have a "memory" for the prior stress, and thus it appears that stress-induced epigenetic marking of the surviving pool of microglia or other factors in the CNS environment or periphery may reprogram the microglia to affect behavioral homeostasis and dysregulation. Repopulated microglia had elevated 2-OH-E production levels that were the same as in microglia of stressed nondepleted animals. In unstressed animals, repopulating microglia do not show a transcriptional profile indicating activation (Huang et al., 2018), but other cells such as astrocytes do show a "cytokine storm" during depletion (Bruttger et al., 2015) that could propagate elevated oxidative stress to repopulating microglia to support dysregulated behavior in previously stressed animals. Alternatively, Weber et al. (2019) found in a similar study of microglial repopulation after repeated social defeat that neurons at $24 \mathrm{~d}$ after repeated defeat retained a sensitization to the prior stressor, suggesting that the altered neuronal state could sensitize new microglia. In our mice, nonmicroglial cells showed ROS activity elevated over that in mice that had not experienced defeat, supporting the likelihood that other cells interact to affect microglial activation status.

It is also possible that enduring signals from the stress-altered periphery and $\mathrm{BBB}$ affect the repopulating microglia. Chronic psychosocial stress concomitantly elevated markers of oxidative stress and inflammation in the brains and plasma of rats (Wilson et al., 2013). Patients with major depressive disorder showed elevated levels of both oxidative stress and inflammation in the blood, which was resolved with antidepressant treatment 

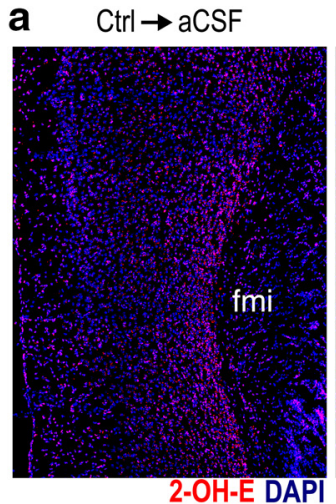

b

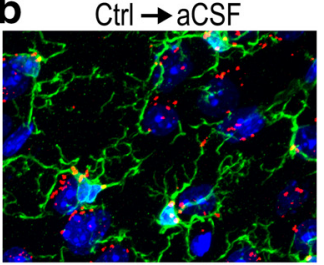

Iba1 2-OH-E DAP

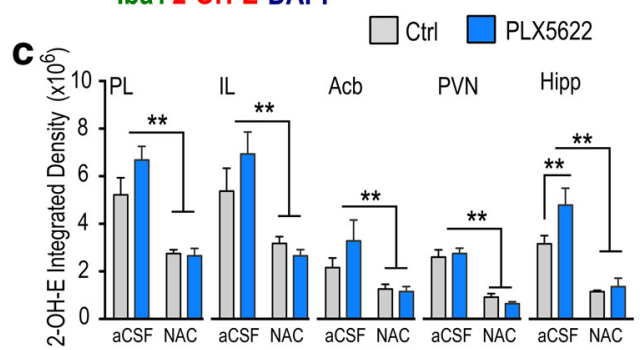

$\mathrm{PLX} 5622 \rightarrow$ aCSF

$\mathrm{PLX} 5622 \rightarrow$ aCSF
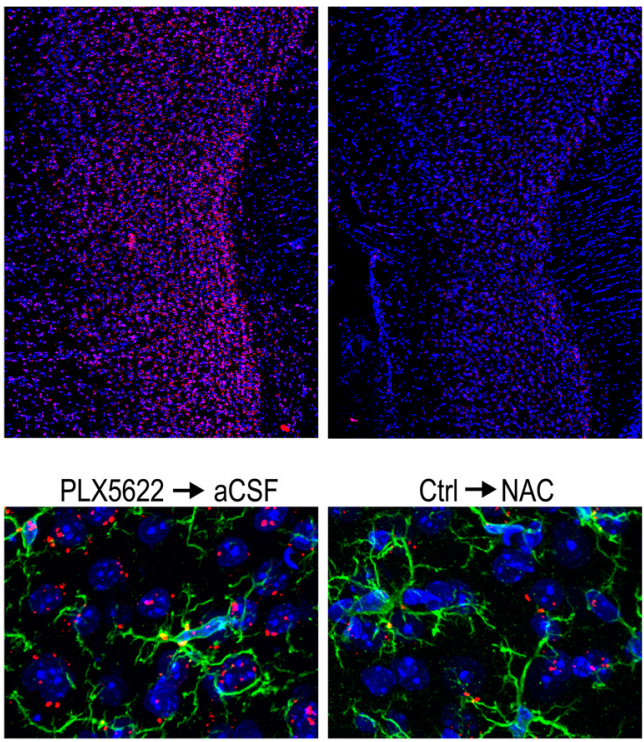

d
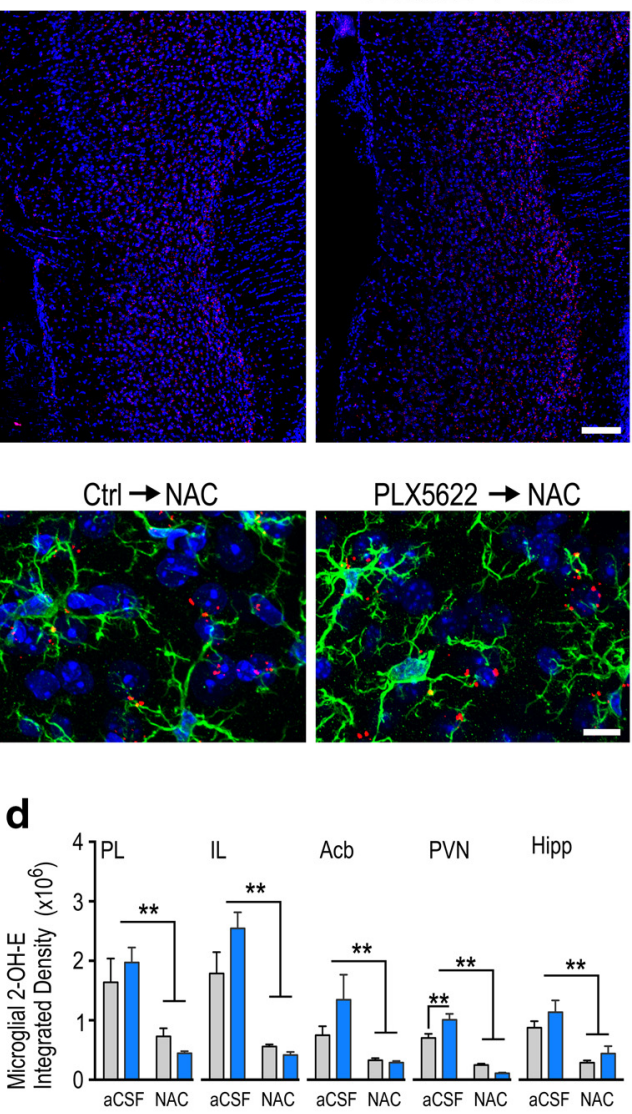

Figure 7. NAC administered during recovery from CSD substantially diminished ROS activity. This effect occurred in mice with intact microglia during stress and in mice in which microglia were ablated during stress but allowed to repopulate during recovery. $\boldsymbol{a}$, Confocal microphotographs show 2-OH-E fluorescence in the mPFC in the four conditions. $\boldsymbol{b}$, Confocal microphotographs show 2-OH-E labeling and Iba1 staining within the PL in the four conditions. $c$, Measures in all cells. $d$, Measures in microglia. ${ }^{* *} p<0.01 . N=6 /$ group. Similar patterns of ROS activity were detected in the five brain regions examined; however, the hippocampus and PVN were more sensitive to prior microglial ablation. Hippocampal cells showed the greatest ROS reactivity to repopulation:2-0H-E fluorescence was significantly elevated in mice treated with PLX5622 during CSD compared with mice with intact microglial populations (effect of PLX treatment: $\left.F_{(1,20)}=4.5, p<0.05\right)$. PVN microglia that repopulated after CSD exhibited significant ROS activity increases compared with microglia intact during CSD (interaction effect: $\left.F_{(1,20)}=12.9, p<0.001\right)$. As in other regions, NAC greatly suppressed ROS activity in both areas. fmi, Forceps minor. Scale bars: $\boldsymbol{a}, 100 \mu \mathrm{m} ; \boldsymbol{b}, 10 \mu \mathrm{m}$.

Experiment 2 (Fig. 3) Can antioxidants prevent behavioral decline during stress?

\begin{tabular}{|c|c|c|c|c|}
\hline NAC & MG $+\mathrm{HC}$ & No $\Delta$ from baseline & Decrease & ic \\
\hline NAC & $\mathrm{MG}+\mathrm{CSD}$ & Behavioral Protection & Decrease & and reduce ROS activity \\
\hline
\end{tabular}

\begin{tabular}{lllll}
\hline Experiment 3 (Fig. 5) Do microglia contribute to behavioral decline during microglial repopulation after stress? \\
MG- & CSD $\rightarrow$ MG+ HC & Behavioral Decline & Increase & $\begin{array}{l}\text { Microglial repopulation after stress } \\
\text { exposure causes behavioral decline } \\
\text { and elevated ROS activity }\end{array}$ \\
MG- & CSD $\rightarrow$ MG- HC & Behavioral Protection & Decrease &
\end{tabular}

Experiment 4 (Figs 6 and 7) Can antioxidants rescue behavioral decline during microglial repopulaton?

\begin{tabular}{|c|c|c|c|c|c|}
\hline MG+ & $\mathrm{CSD} \rightarrow$ & $\mathrm{MG}+\mathrm{HC}$ & Behavioral Protection & Decrease & Antioxidants rescue behavior during \\
\hline MG- & $\mathrm{CSD} \Rightarrow$ & $\mathrm{MG}+\mathrm{HC}$ & Behavioral Protection & Decrease & previously stressed mice \\
\hline
\end{tabular}

Figure 8. The main conditions, findings, and conclusions are provided for each experiment. MG ${ }^{-}$, microglia depleted; $M G^{+}$(shaded green), microglia present or repopulated; NAC intracerebroventricular $\mathrm{N}$-acetylcysteine treatment (boxed in blue, indicating period of drug administration).

(Rawdin et al., 2013). Thus, peripheral as well as central ROS/ inflammation mechanisms may contribute to the adverse effects of CSD. Chronic stress and depression affect cerebrovascular function (Burrage et al., 2018), and we previously determined that microglia are sensitive to severe reductions in BBB integrity caused by CSD (Lehmann et al., 2018). Alterations in brain microenvironments that persist during stress recovery may underlie some of the effects observed in the repopulation studies. Notably, 
elevated ROS activity is particularly damaging to the neurovascular environment that supports blood-brain barrier integrity (Carvalho and Moreira, 2018). We are currently exploring events associated with the repair of damage to the BBB caused by CSD and suspect that microglia are activated in this process.

One unresolved question is the cause of the exaggerated elevation in ROS activity in the PL and IL areas of the MPFC, a key station for emotional processing (Price and Drevets, 2012). Microglial activity may be associated with synaptic pruning, which might be preferentially ongoing in this limbic area known to modulate emotional responses to stress (Schafer et al., 2012; Hinwood et al., 2013; Calcia et al., 2016; Wohleb et al., 2018), or with CSD-induced demyelination (Lehmann et al., 2017), possibly resulting from microglia-oligodendrocyte interactions (Peferoen et al., 2014). Understanding of the anatomical selectivity of heightened microglial and ROS activity in the mPFC is currently lacking.

In summary, we previously examined the effects of CSD on microglial activity and found a correlation between microglial gene expression profiles and susceptibility to CSD effects: susceptible (showing anxiety and social deficits) but not resilient mice had microglial gene expression representing inflammation, phagocytosis, blood-brain barrier breakdown, and elevated 2-OH-E production (Lehmann et al., 2018). Based on these findings, we focused on microglia and oxidative stress as key factors. The data showed that in all conditions, elevated ROS production in the presence of microglia was associated with stress-induced anxiety and antisocial behavior, whereas without microglia or oxidative stress, behavioral protection prevailed (Fig. 8). The data suggest that microglia-dependent ROS production is a major way in which microglia affect the CNS environment that supports stress circuitry. However, the NAC-induced ROS inhibition was in all cells and not just microglia, so at this point, we conclude that both microglia and overall CNS ROS activity are linked and are both causally involved in producing adverse stress effects on behavior. Studies in which ROS activity is selectively blocked in microglia will address whether such a targeted manipulation would be sufficient to protect against chronic psychosocial stress effects.

\section{References}

Ajami B, Bennett JL, Krieger C, Tetzlaff W, Rossi FM (2007) Local selfrenewal can sustain CNS microglia maintenance and function throughout adult life. Nat Neurosci 10:1538-1543.

Bavarsad Shahripour R, Harrigan MR, Alexandrov AV (2014) N-acetylcysteine (NAC) in neurological disorders: mechanisms of action and therapeutic opportunities. Brain Behav 4:108-122.

Bennett ML, Bennett FC, Liddelow SA, Ajami B, Zamanian JL, Fernhoff NB, Mulinyawe SB, Bohlen CJ, Adil A, Tucker A, Weissman IL, Chang EF, Li G, Grant GA, Hayden Gephart MG, Barres BA (2016) New tools for studying microglia in the mouse and human CNS. Proc Natl Acad Sci U S A 113:E1738-E1746.

Black CN, Bot M, Scheffer PG, Cuijpers P, Penninx BW (2015) Is depression associated with increased oxidative stress? A systematic review and metaanalysis. Psychoneuroendocrinology 51:164-175.

Bruttger J, Karram K, Wörtge S, Regen T, Marini F, Hoppmann N, Klein M, Blank T, Yona S, Wolf Y, Mack M, Pinteaux E, Müller W, Zipp F, Binder H, Bopp T, Prinz M, Jung S, Waisman A (2015) Genetic cell ablation reveals clusters of local self-renewing microglia in the mammalian central nervous system. Immunity 43:92-106.

Burrage E, Marshall KL, Santanam N, Chantler PD (2018) Cerebrovascular dysfunction with stress and depression. Brain Circ 4:43-53.

Butovsky O, Weiner HL (2018) Microglial signatures and their role in health and disease. Nat Rev Neurosci 19:622-635.

Calcia MA, Bonsall DR, Bloomfield PS, Selvaraj S, Barichello T, Howes OD (2016) Stress and neuroinflammation: a systematic review of the effects of stress on microglia and the implications for mental illness. Psychopharmacology (Berl) 233:1637-1650.

Carvalho C, Moreira PI (2018) Oxidative stress: a major player in cerebrovascular alterations associated to neurodegenerative events. Front Physiol 9:806.

Cheng G, Zielonka M, Dranka B, Kumar SN, Myers CR, Bennett B, Garces AM, Dias Duarte Machado LG, Thiebaut D, Ouari O, Hardy M, Zielonka J, Kalyanaraman B (2018) Detection of mitochondria-generated reactive oxygen species in cells using multiple probes and methods: potentials, pitfalls, and the future. J Biol Chem 293:10363-10380.

Cobley JN, Fiorello ML, Bailey DM (2018) 13 reasons why the brain is susceptible to oxidative stress. Redox Biol 15:490-503.

Dagher NN, Najafi AR, Kayala KM, Elmore MR, White TE, Medeiros R, West BL, Green KN (2015) Colony-stimulating factor 1 receptor inhibition prevents microglial plaque association and improves cognition in 3xTg-AD mice. J Neuroinflammation 12:139.

ElAli A, Rivest S (2016) Microglia ontology and signaling. Front Cell Dev Biol 4:72.

Elmore MR, Najafi AR, Koike MA, Dagher NN, Spangenberg EE, Rice RA, Kitazawa M, Matusow B, Nguyen H, West BL, Green KN (2014) Colony-stimulating factor 1 receptor signaling is necessary for microglia viability, unmasking a microglia progenitor cell in the adult brain. Neuron 82:380-397.

Elmore MR, Lee RJ, West BL, Green KN (2015) Characterizing newly repopulated microglia in the adult mouse: impacts on animal behavior, cell morphology, and neuroinflammation. PLoS One 10:e0122912.

Epelman S, Lavine KJ, Randolph GJ (2014) Origin and functions of tissue macrophages. Immunity 41:21-35.

Finkel T (2012) Signal transduction by mitochondrial oxidants. J Biol Chem 287:4434-4440.

Forkink M, Smeitink JA, Brock R, Willems PH, Koopman WJ (2010) Detection and manipulation of mitochondrial reactive oxygen species in mammalian cells. Biochim Biophys Acta 1797:1034-1044.

Frank MG, Weber MD, Watkins LR, Maier SF (2015) Stress sounds the alarmin: the role of the danger-associated molecular pattern HMGB1 in stress-induced neuroinflammatory priming. Brain Behav Immun 48:1-7.

Ginhoux F, Greter M, Leboeuf M, Nandi S, See P, Gokhan S, Mehler MF, Conway SJ, Ng LG, Stanley ER, Samokhvalov IM, Merad M (2010) Fate mapping analysis reveals that adult microglia derive from primitive macrophages. Science 330:841-845.

Hammond TR, Robinton D, Stevens B (2018) Microglia and the brain: complementary partners in development and disease. Annu Rev Cell Dev Biol 34:523-544.

Hinwood M, Tynan RJ, Charnley JL, Beynon SB, Day TA, Walker FR (2013) Chronic stress induced remodeling of the prefrontal cortex: structural re-organization of microglia and the inhibitory effect of minocycline. Cereb Cortex 23:1784-1797.

Huang Y, Xu Z, Xiong S, Sun F, Qin G, Hu G, Wang J, Zhao L, Liang YX, Wu T, Lu Z, Humayun MS, So KF, Pan Y, Li N, Yuan TF, Rao Y, Peng B (2018) Repopulated microglia are solely derived from the proliferation of residual microglia after acute depletion. Nat Neurosci 21:530-540.

Ibi M, Liu J, Arakawa N, Kitaoka S, Kawaji A, Matsuda KI, Iwata K, Matsumoto M, Katsuyama M, Zhu K, Teramukai S, Furuyashiki T, YabeNishimura C (2017) Depressive-like behaviors are regulated by NOX1/ NADPH oxidase by redox modification of NMDA receptor 1 . J Neurosci 37:4200-4212.

Kendler KS, Gardner CO (2010) Dependent stressful life events and prior depressive episodes in the prediction of major depression: the problem of causal inference in psychiatric epidemiology. Arch Gen Psychiatry 67: $1120-1127$

Keren-Shaul H, Spinrad A, Weiner A, Matcovitch-Natan O, Dvir-Szternfeld R, Ulland TK, David E, Baruch K, Lara-Astaiso D, Toth B, Itzkovitz S, Colonna M, Schwartz M, Amit I (2017) A unique microglia type associated with restricting development of Alzheimer's disease. Cell 169:12761290.e17.

Lambeth JD (2004) NOX enzymes and the biology of reactive oxygen. Nat Rev Immunol 4:181-189.

Lawson LJ, Perry VH, Dri P, Gordon S (1990) Heterogeneity in the distribution and morphology of microglia in the normal adult mouse brain. Neuroscience 39:151-170.

Lehmann ML, Herkenham M (2011) Environmental enrichment confers 
stress resiliency to social defeat through an infralimbic cortex-dependent neuroanatomical pathway. J Neurosci 31:6159-6173.

Lehmann ML, Cooper HA, Maric D, Herkenham M (2016) Social defeat induces depressive-like states and microglial activation without involvement of peripheral macrophages. J Neuroinflammation 13:224.

Lehmann ML, Weigel TK, Elkahloun AG, Herkenham M (2017) Chronic social defeat reduces myelination in the mouse medial prefrontal cortex. Sci Rep 7:46548.

Lehmann ML, Weigel TK, Cooper HA, Elkahloun AG, Kigar SL, Herkenham M (2018) Decoding microglia responses to psychosocial stress reveals blood-brain barrier breakdown that may drive stress susceptibility. Sci Rep 8:11240.

Liu R, Liu IY, Bi X, Thompson RF, Doctrow SR, Malfroy B, Baudry M (2003) Reversal of age-related learning deficits and brain oxidative stress in mice with superoxide dismutase/catalase mimetics. Proc Natl Acad Sci U S A 100:8526-8531.

McKim DB, Weber MD, Niraula A, Sawicki CM, Liu X, Jarrett BL, RamirezChan K, Wang Y, Roeth RM, Sucaldito AD, Sobol CG, Quan N, Sheridan JF, Godbout JP (2018) Microglial recruitment of IL-1 $\beta$-producing monocytes to brain endothelium causes stress-induced anxiety. Mol Psychiatry 23:1421-1431.

Menard C, Pfau ML, Hodes GE, Kana V, Wang VX, Bouchard S, Takahashi A, Flanigan ME, Aleyasin H, LeClair KB, Janssen WG, Labonté B, Parise EM, Lorsch ZS, Golden SA, Heshmati M, Tamminga C, Turecki G, Campbell M, Fayad ZA, et al. (2017) Social stress induces neurovascular pathology promoting depression. Nat Neurosci 20:1752-1760.

Minakami R, Sumimotoa H (2006) Phagocytosis-coupled activation of the superoxide-producing phagocyte oxidase, a member of the NADPH oxidase (nox) family. Int J Hematol 84:193-198.

Nazarewicz RR, Bikineyeva A, Dikalov SI (2013) Rapid and specific measurements of superoxide using fluorescence spectroscopy. J Biomol Screen 18:498-503.

Ng F, Berk M, Dean O, Bush AI (2008) Oxidative stress in psychiatric disorders: evidence base and therapeutic implications. Int J Neuropsychopharmacol 11:851-876.

Nimmerjahn A, Kirchhoff F, Helmchen F (2005) Resting microglial cells are highly dynamic surveillants of brain parenchyma in vivo. Science 308:1314-1318.

Peferoen L, Kipp M, van der Valk P, van Noort JM, Amor S (2014) Oligodendrocyte-microglia cross-talk in the central nervous system. Immunology 141:302-313.

Picard M, McEwen BS (2018) Psychological stress and mitochondria: a systematic review. Psychosom Med 80:141-153.

Pollak DD, Rey CE, Monje FJ (2010) Rodent models in depression research: classical strategies and new directions. Ann Med 42:252-264.

Popa-Wagner A, Mitran S, Sivanesan S, Chang E, Buga AM (2013) ROS and brain diseases: the good, the bad, and the ugly. Oxid Med Cell Longev 2013:963520.

Price JL, Drevets WC (2012) Neural circuits underlying the pathophysiology of mood disorders. Trends Cogn Sci 16:61-71.

Prinz M, Priller J (2014) Microglia and brain macrophages in the molecular age: from origin to neuropsychiatric disease. Nat Rev Neurosci 15: $300-312$.

Prinz M, Tay TL, Wolf Y, Jung S (2014) Microglia: unique and common features with other tissue macrophages. Acta Neuropathol 128:319-331.

Quinn MT, Ammons MC, Deleo FR (2006) The expanding role of NADPH oxidases in health and disease: no longer just agents of death and destruction. Clin Sci (Lond) 111:1-20.

Rammal H, Bouayed J, Younos C, Soulimani R (2008) Evidence that oxidative stress is linked to anxiety-related behaviour in mice. Brain Behav Immun 22:1156-1159.

Rawdin BJ, Mellon SH, Dhabhar FS, Epel ES, Puterman E, Su Y, Burke HM, Reus VI, Rosser R, Hamilton SP, Nelson JC, Wolkowitz OM (2013) Dysregulated relationship of inflammation and oxidative stress in major depression. Brain Behav Immun 31:143-152.

Robinson KM, Janes MS, Pehar M, Monette JS, Ross MF, Hagen TM, Murphy MP, Beckman JS (2006) Selective fluorescent imaging of superoxide in vivo using ethidium-based probes. Proc Natl Acad Sci U S A 103: $15038-15043$.
Rojo AI, McBean G, Cindric M, Egea J, López MG, Rada P, Zarkovic N, Cuadrado A (2014) Redox control of microglial function: molecular mechanisms and functional significance. Antioxid Redox Signal 21: $1766-1801$.

Salim S (2014) Oxidative stress and psychological disorders. Curr Neuropharmacol 12:140-147.

Sapolsky RM (1999) Glucocorticoids, stress, and their adverse neurological effects: relevance to aging. Exp Gerontol 34:721-732.

Schafer DP, Lehrman EK, Kautzman AG, Koyama R, Mardinly AR, Yamasaki R, Ransohoff RM, Greenberg ME, Barres BA, Stevens B (2012) Microglia sculpt postnatal neural circuits in an activity and complementdependent manner. Neuron 74:691-705.

Schiavone S, Sorce S, Dubois-Dauphin M, Jaquet V, Colaianna M, Zotti M, Cuomo V, Trabace L, Krause KH (2009) Involvement of NOX2 in the development of behavioral and pathologic alterations in isolated rats. Biol Psychiatry 66:384-392.

Schiavone S, Jaquet V, Trabace L, Krause KH (2013) Severe life stress and oxidative stress in the brain: from animal models to human pathology. Antioxid Redox Signal 18:1475-1490.

Seo JS, Park JY, Choi J, Kim TK, Shin JH, Lee JK, Han PL (2012) NADPH oxidase mediates depressive behavior induced by chronic stress in mice. J Neurosci 32:9690-9699.

Sheffner AL, Medler EM, Bailey KR, Gallo DG, Mueller AJ, Sarett HP (1966) Metabolic studies with acetylcysteine. Biochem Pharmacol 15:1523-1535.

Sorrells SF, Caso JR, Munhoz CD, Sapolsky RM (2009) The stressed CNS: when glucocorticoids aggravate inflammation. Neuron 64:33-39.

Tay TL, Savage JC, Hui CW, Bisht K, Tremblay MÈ (2017a) Microglia across the lifespan: from origin to function in brain development, plasticity and cognition. J Physiol 595:1929-1945.

Tay TL, Mai D, Dautzenberg J, Fernández-Klett F, Lin G, Sagar, Datta M, Drougard A, Stempfl T, Ardura-Fabregat A, Staszewski O, Margineanu A, Sporbert A, Steinmetz LM, Pospisilik JA, Jung S, Priller J, Grün D, Ronneberger O, Prinz M (2017b) A new fate mapping system reveals context-dependent random or clonal expansion of microglia. Nat Neurosci 20:793-803.

Turrens JF (2003) Mitochondrial formation of reactive oxygen species. J Physiol 552:335-344.

Tynan RJ, Naicker S, Hinwood M, Nalivaiko E, Buller KM, Pow DV, Day TA, Walker FR (2010) Chronic stress alters the density and morphology of microglia in a subset of stress-responsive brain regions. Brain Behav Immun 24:1058-1068.

Walker FR, Nilsson M, Jones K (2013) Acute and chronic stress-induced disturbances of microglial plasticity, phenotype and function. Curr Drug Targets 14:1262-1276.

Weber MD, McKim DB, Niraula A, Witcher KG, Yin W, Sobol CG, Wang Y, Sawicki CM, Sheridan JF, Godbout JP (2019) The influence of microglial elimination and repopulation on stress sensitization induced by repeated social defeat. Biol Psychiatry 85:667-678.

Wilson CB, McLaughlin LD, Nair A, Ebenezer PJ, Dange R, Francis J (2013) Inflammation and oxidative stress are elevated in the brain, blood, and adrenal glands during the progression of post-traumatic stress disorder in a predator exposure animal model. PLoS One 8:e76146.

Wohleb ES, McKim DB, Sheridan JF, Godbout JP (2014) Monocyte trafficking to the brain with stress and inflammation: a novel axis of immuneto-brain communication that influences mood and behavior. Front Neurosci 8:447.

Wohleb ES, Terwilliger R, Duman CH, Duman RS (2018) Stress-induced neuronal colony stimulating factor 1 provokes microglia-mediated neuronal remodeling and depressive-like behavior. Biol Psychiatry 83:38-49.

Zafir A, Banu N (2009) Modulation of in vivo oxidative status by exogenous corticosterone and restraint stress in rats. Stress 12:167-177.

Zhao H, Joseph J, Fales HM, Sokoloski EA, Levine RL, Vasquez-Vivar J, Kalyanaraman B (2005) Detection and characterization of the product of hydroethidine and intracellular superoxide by HPLC and limitations of fluorescence. Proc Natl Acad Sci U S A 102:5727-5732.

Zielonka J, Vasquez-Vivar J, Kalyanaraman B (2008) Detection of 2-hydroxyethidium in cellular systems: a unique marker product of superoxide and hydroethidine. Nat Protoc 3:8-21. 